\title{
A case of occult colon cancer with sepsis as the primary manifestation guided by bone marrow puncture
}

hua jun wang ( $\nabla$ whj269696@163.com )

ning bo shi yin zhou ren min yi yuan: Yinzhou People's Hospital

chenjie ZHOU

ning bo shi yin zhou ren min yi yuan: Yinzhou People's Hospital

jianhua Wang

ning bo shi yin zhou ren min yi yuan: Yinzhou People's Hospital

\section{Case report}

Keywords: occult colon cancer, Bone marrow metastasis, thrombocytopenia

Posted Date: April 2nd, 2021

DOI: https://doi.org/10.21203/rs.3.rs-312591/v1

License: (c) (i) This work is licensed under a Creative Commons Attribution 4.0 International License.

Read Full License 


\section{Abstract}

Background: Bone marrow metastasis is common in liver cancer and lung cancer, but there are few reports on bone marrow metastasis of colon cancer. So far, there are no research reports from mainland China; especially reports of bone marrow metastasis combined with septic shock as the main manifestation are even rarer.

Case presentation: A 71-year-old elderly woman with sepsis as the first symptom, mainly manifested as high fever, low blood pressure, high inflammation indicators, etc. CT examination revealed mild inflammation of the lungs and no obvious abnormalities in the abdomen. Blood culture culture suggested Escherichia coli and Aeromonas hydrophila/caviae. After anti-infective treatment, the patient's sepsis symptoms improved significantly, but after repeated platelet transfusion, he still could not correct hypothrombosis. Finally, a large number of malignant cells were found through a bone marrow puncture smear, and further PET-CT examination confirmed that the ascending colon malignant tumor was accompanied by multiple metastases such as the liver and bones. The patient was eventually unable to tolerate surgery due to bone marrow failure and liver failure and died 3 weeks after admission.

Conclusions: We hope that this case report can raise people's awareness of the insidiousness of colon cancer and the possibility of metastasis to the bone marrow, especially for patients with progressive thrombocytopenia that is difficult to correct. Thrombocytopenia needs to be treated differently from sepsis.

\section{Case Presentation}

The patient was a 71-year-old female with hypertension for 3 years. She received oral $80 \mathrm{mg}$ Jiesartan capsules daily to control blood pressure, and her blood pressure was controlled. The patient developed fever 3 hours prior to admission, with a peak temperature of $39^{\circ} \mathrm{C}$, accompanied by chills, no cough, expectoration, pharyngeal pain, abdominal pain, diarrhea, increased urination frequency, increase urination urgency, perianal pain and other symptoms. Routine blood examination showed the following: white blood cell count $13.2 \times 10^{\wedge} 9 / \mathrm{L}$, neutrophil percentage $88.5 \%$, hemoglobin $66 \mathrm{~g} / \mathrm{L}$, platelet count $11 \times 10^{\wedge} 9 / \mathrm{L}$, and blood glucose $33 \mathrm{mmol} / \mathrm{L}$. The chest and abdomen CT findings were as follows: 1 . mild inflammationin both lungs; 2 . bilateral pleural thickening. The suspected diagnoseson admission were as follows: 1. fever of undetermined origin (potentially indicating severe sepsis); 2.pneumonia; and 3. hypertension. After admission, the patientwas treated with intravenous melopepenan 0.5 Q6h as antiinflammatory therapy, intravenous human immunoglobulin $5 \mathrm{~g}$ QD to improve immunity, 4 units of erythrocyte suspension corrects anemia, and 10 units of platelets corrects low platelets. In addition, insulin micropumps were continuously used to control blood glucose. The next day, the patient was cold and shivering, the temperature reached $40^{\circ} \mathrm{C}$, and the blood pressure dropped to $85 / 53 \mathrm{mmHg}$. The telephone report from the microbiology unitindicated that Escherichia coli and Aeromonas hydrophila/caviae were identified from the blood culture. The patient was transferred to the ICU, and a Meropeian 0.5micropump (Q6h) combined with $50 \mathrm{mg}$ intravenous teglocytin Q12h was used to fight 
infection, fluid resuscitation, vasoactive drugs maintain blood pressure stability.. Recombinant human granulocyte colony-stimulating factor was used to elevate the white blood cell count, and recombinant human platelet growth factor was used to promote platelet production. In addition, erythrocyte suspensions, platelets, and other supportive treatments were administered. One week after the antiinfection treatment, the patient's infection was controlled, the blood pressure and body temperature returned to normal, and the blood culture was negative, but the platelet count was difficult to maintain (Fig. 1). A bone marrow biopsy was performed. The bone marrow smear showed many malignant cells (Fig. 2). Under suspicion that the cancer that had spread to the bone marrow, further whole-body ${ }^{18} \mathrm{~F}-\mathrm{FDG}$ PET/CT examination was performed, and the results revealed high metabolic signals in the ascending colon (Fig. 3). An ascending colon malignant tumor was suspected. Because the patient suffered from thrombocytopenia as a result of the bone marrow metastasis, surgery and endoscopy were contraindicated;eventually, the tumor progressed, and the patient died 3 weeks later.

\section{Discussion}

Bone marrow-metastatic carcinoma results from the migration of medullary tumor cells to the bone marrow through blood or body fluids. Bone marrow metastasis can occur with almost all malignant tumors, of which lung cancer, breast cancer and prostate cancer are the most prone to bone marrow metastasis, while colon cancer is relatively unlikely to lead to bone marrow metastasis[1, 2]. In addition to the clinical symptoms of primary tumors, bone marrow metastasis is generally characterized by an abnormal blood system. This dysfunction of the blood system mainly manifests as myelosuppression,including decreased counts of platelets, hemoglobin level and white blood cell counts, and some patients have myeloproliferative manifestations [3, 4]. When the patient was admitted to the hospital, there were no obvious other clinical symptoms, the tumor markers were normal, and no special abnormalities were found on chest and abdomen CT imaging; this lack of symptoms was consistent with the early stage of colon cancer. In this case, severe sepsis was the primary manifestation, including high fever, shock, and detection of Escherichia coli and Aeromonashydrophila/caviae on blood culture. The sepsis may have been a result of the patient's tumor destroying the normal mucosal barrier of the intestinal wall, which allowed the intestinal flora to enter into the blood; such a manifestation has rarely been reported in previous cases. Whether trilineage decreases in blood cells are direct result of hematological disease or secondary to bone marrow suppression caused by the severe sepsis is often difficult to determine at first, which can easily result in missed or misdiagnosed cases. Thrombocytopenia caused by sepsis is generally related to bone marrow suppression and increased destruction. With infection control, the platelet count gradually returns to normal within 6 days, especially after the application of thrombopoietin[5]. However, when the infection was controlled and the blood culture turned negative, the platelet count was difficult to maintain even after repeated platelet transfusions. A bone marrow puncture was further performed, and the bone marrow smear revealed a large number of malignant cells, which further confirmed the diagnosis of colon cancer suggested by the systemic ${ }^{18} \mathrm{~F}$ FDG PET/CT examination. Therefore, bone marrow smears have important auxiliary value for the diagnosis of some occult tumors.Studies have shown that $41 \%$ of patients with malignant tumors have 
bone marrow metastasis, and evaluation of bone marrow smears to identify cancer cells is important for the diagnosis of bone marrow-metastatic cancer. Finding cancer cells on bone marrow smears or in biopsy samples is helpful for the diagnosis of tumors, and some unique cells are even helpful for identifying the primary tumor origin[6, 7]. It is worth pointing out that not all metastatic cancers can be found by a single bone marrow biopsy, and staining the sediment of the puncture or bone marrow biopsy samples is necessary for improving the positive diagnosis rate [8]. The diagnosis of bone marrow metastasis can assist the diagnosis of primary tumors and prognostication, suggesting that it is a useful auxiliary method for the diagnosis of tumors.

\section{Conclusions}

Colon cancer with sepsis as the first manifestation is very rare. When the progressive reduction of platelets is difficult to correct, it is necessary to consider tumor bone marrow metastasis, especially when sepsis has been controlled. At this time, At this time, bone marrow aspiration or biopsy and PET-CT can help confirm the diagnosis of occult colon cancer.

\section{Declarations}

\section{Ethical approval and consent to participate:}

All procedures performed in studies involving human participants were performed in accordance with the ethical standards of the Ethics Committee of Ningbo Yinzhou People's Hospital and the 1964 Declaration of Helsinki and its later amendments or comparable ethical standards.Informed consent was obtained from the patient

Consent for publication:Written informed consent was obtained from the patient for publication of this case report and any accompanying images. A copy of the written consent form is available for review by the Editor-in-Chief of this journal.

Availability of data and materials:All data have been presented within the manuscript and in the form of images.

Competing Interests:We declare have no competing interests to declare.

Funding:No funding was received for this study.

\section{Authors' Contributions:}

GC was responsible for preparing the paper and providedthe final approval. CZ was responsible for the acquisition, analysis, or interpretation of data and revised the paper critically for important intellectual content. FY and YLN were responsible for polishing the English translation. All authors have read and approved the manuscript. 
Acknowledgments:

We would like to thank the patient for agreeing to the publication of this article and all the doctors and staff who assisted us with this report. We are also very grateful to the other translators and indirect tutors.

\section{References}

1. Lim DH, Lee SI, Park KW. Bone marrow metastasis of colon cancer as the first site of recurrence: a case report. Oncol Lett. 2014;8:2672-4. 10.3892/ol.2014.2581.

2. Chuwa H, Kassam NM, Wambura C, Sherman OA, Surani S. Disseminated Carcinomatosis of Bone Marrow in an African Man with Metastatic Descending Colon Carcinoma. Cureus. $2020 \mathrm{Apr}$ 8;12(4):e7593. doi: 10.7759/cureus.7593. PMID: 32399327; PMCID: PMC7212736.

3. Tyagi R, Singh A, Garg B, Sood N. Beware of Bone Marrow: Incidental Detection and Primary Diagnosis of Solid Tumours in Bone Marrow Aspiration and Biopsies; A Study of 22 Cases. Iran J Pathol. 2018;13(1):78-84.

4. Lai GM, Lin J-T, Chang C-S. Metastatic bone marrow tumors manifested by hematologic disorders: study of thirty-four cases and review of literature. J Anal Oncol. 2014;3(4):185-90.

5. Wu Q, Ren J, Wu X, et al. Recombinant human thrombopoietin improves platelet counts and reduces platelet transfusion possibility among patients with severe sepsis and thrombocytopenia: a prospective study. J Crit Care. 2014 Jun;29(3):362-6. doi: 10.1016/j.jcrc.2013.11.023. Epub 2013 Dec 12. PMID: 24405656.

6. Chen Guiling Z. Ruibo. Analysis of the role of bone marrow biopsy and smear in the diagnosis of bone marrow metastasis [J]. Chinese Journal of Cancer Prevention Treatment. 2016;S2:271-2.

7. Kucukzeybek BB, Calli AO, Kucukzeybek Y, et al. The prognostic significance of bone marrow metastases: evaluation of 58 cases. Indian J Pathol Microbiol. 2014 Jul-Sep;57(3):396-9. doi: 10.4103/0377-4929.138728. PMID: 25118730.

8. Guan JH, Wang XN, Ma K. One-step method of bone marrow aspiration and biopsy applied in diagnosis of the bone marrow metastatic cancer. Zhongguo Shi Yan Xue Ye Xue Za Zhi. 2013 Aug;21(4):1054-7. Chinese. doi: 10.7534/j.issn.1009-2137.2013.04.046. PMID: 23998611.

\section{Figures}




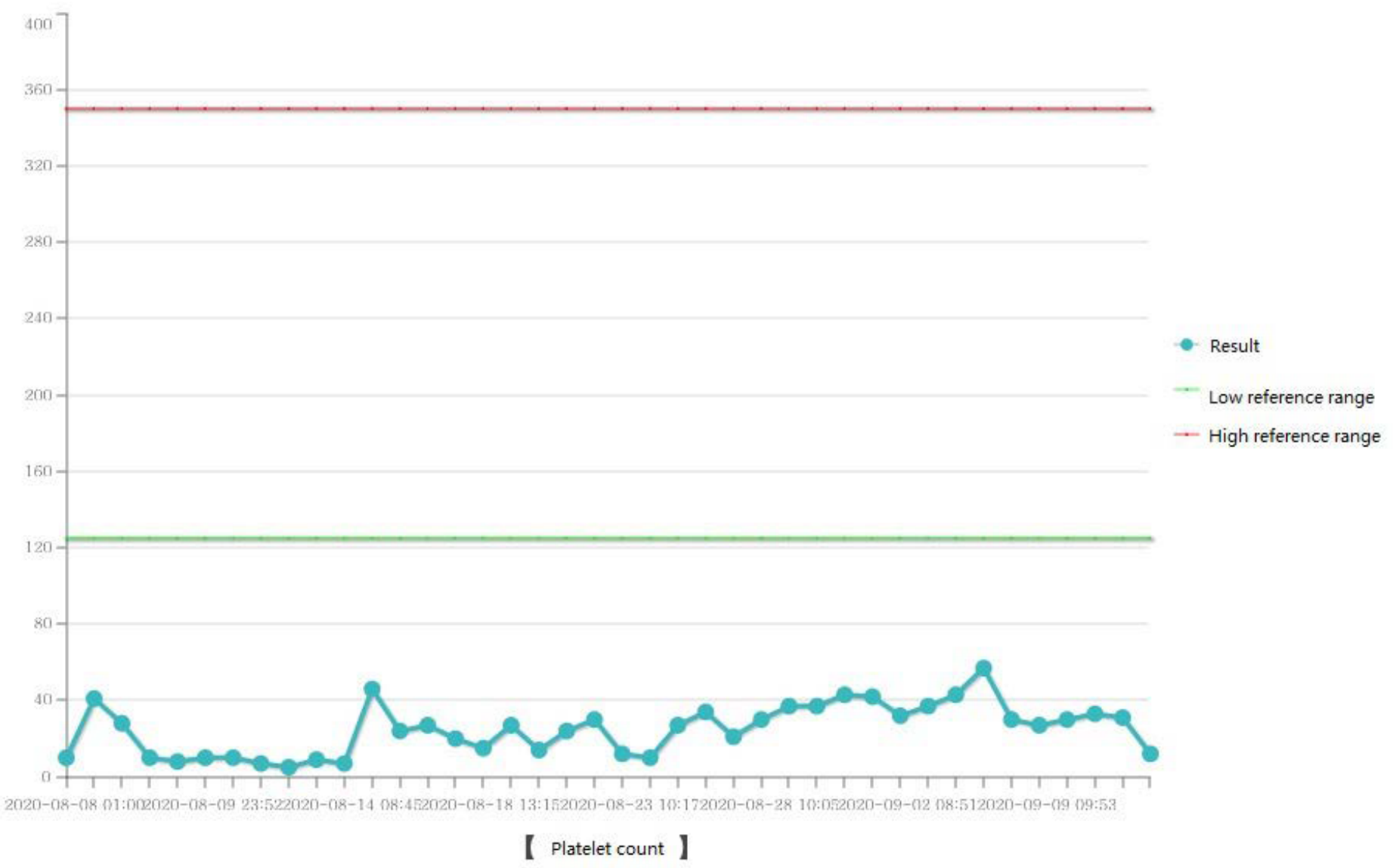

Figure 1

Platelets were maintained at low levels even after repeated transfusions

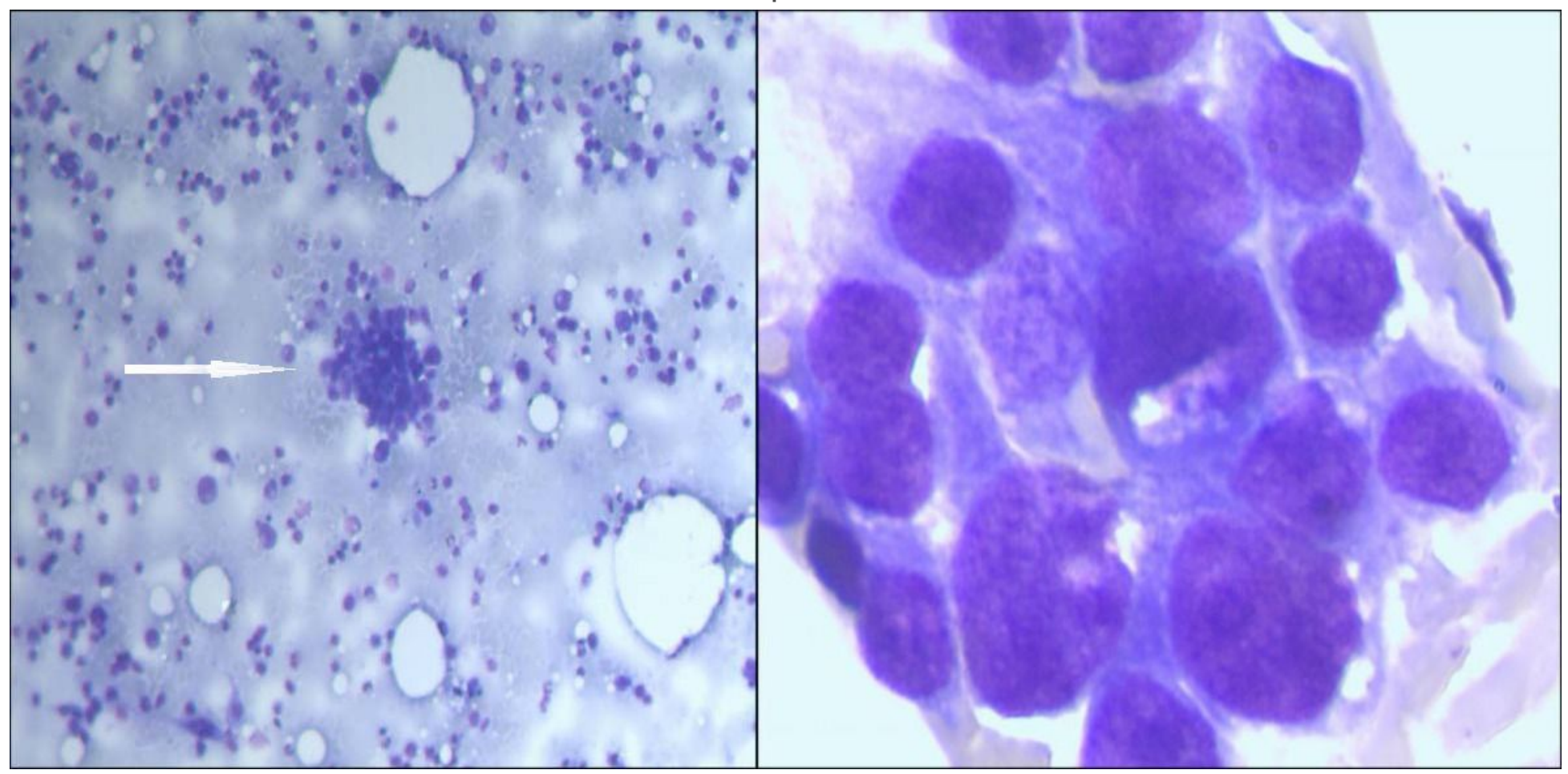


Figure 2

Left panel: $a \rrbracket$ The bone marrow smear is stained with Wright Jim can be found a class of abnormal

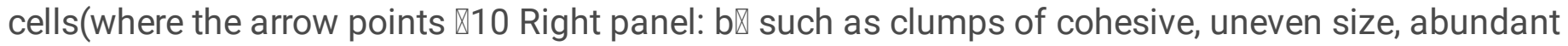
cytoplasm, irregular margins, rough chromatin, visible with 1-3 faint nucleoli( $(100)$
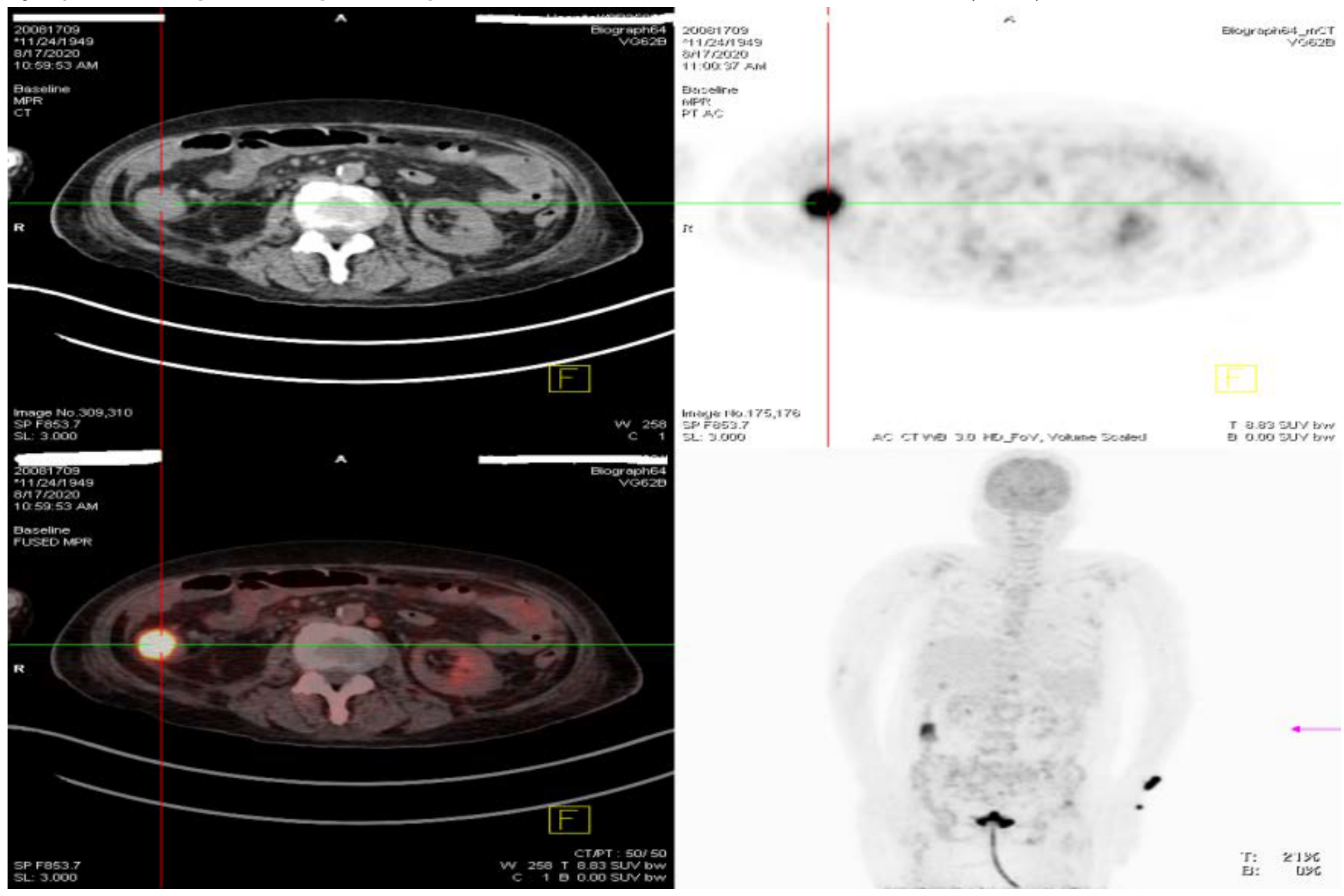

\section{Figure 3}

18F-FDG PET/CT showed abnormally high signals in ascending colon and left radius 